\title{
Effect of a Negative Poisson Ratio in the Tension of Ceramics
}

\author{
Fan Song, ${ }^{1,2, *}$ Junbing Zhou, ${ }^{1}$ Xianghong Xu, ${ }^{1}$ Yi Xu, ${ }^{1}$ and Yilong Bai ${ }^{1}$ \\ ${ }^{1}$ State Key Laboratory of Nonlinear Mechanics (LNM), Institute of Mechanics, Chinese Academy of Sciences, \\ Beijing 100080, People's Republic of China \\ ${ }^{2}$ Center for Biomechanics and Bioengineering (CBB), Institute of Mechanics, Chinese Academy of Sciences, \\ Beijing 100080, People's Republic of China \\ (Received 30 October 2007; published 18 June 2008)
}

\begin{abstract}
The effect of a negative Poisson ratio is experimentally revealed in the tension deformation of a natural layered ceramic. This effect can increase the volume strain energy per unit volume by $1100 \%$ and, simultaneously, decrease the deformation strain energy per unit volume by about $44 \%$, so that it effectively enhances the deformation capacity by about 1 order of magnitude in the tension of the material. The present study also shows that the physical mechanisms producing the effect are attributed to the climbing on one another of the nanostructures in the natural material, which provides a guide to the design of synthetic toughening composites.
\end{abstract}

DOI: 10.1103/PhysRevLett.100.245502

PACS numbers: $62.20 . \mathrm{F}-$, 46.50.+a, 81.07. $-\mathrm{b}, 87.85 . \mathrm{J}-$

Poisson's ratio is defined as the negative of the lateral strain divided by the longitudinal strain when a load is applied in the longitudinal direction. It theoretically ranges from -1.0 to 0.5 based on thermodynamic considerations of strain energy in the theory of elasticity [1]. In practice, all ordinary materials, which undergo a lateral contraction when stretched and a lateral expansion when compressed, exhibit a positive Poisson ratio from 0 to 0.5 [2]. For example, the Poisson ratios for various materials are approximately zero for cork, $0.14 \sim 0.17$ for $\mathrm{SiC}, 0.26$ for $\mathrm{Al}_{2} \mathrm{O}_{3}, 0.33$ for aluminum, 0.42 for silicon, 0.45 for lead, and nearly 0.5 for rubbers and soft biological tissues [3-6]. The materials with a negative Poisson ratio are theoretically permissible but, with a few exceptions, have not been observed in real materials and are believed to be unknown $[2,3]$. However, Love presented a single example of cubic "single crystal" pyrite with a negative Poison's ratio of -0.14 and suggested that the effect may result from a twinned crystal [7]. Recently, it was indicated that negative Poisson's ratios may be a feature of cubic metals $[8,9]$. In addition, Lakes obtained a type of foam material with a negative Poisson's ratio by designing cellular structures [3] and he pointed out that the effect of a negative Poisson's ratio may play an important role in the fracture toughness of the materials $[10,11]$. In particular, the man-made hierarchical laminates with a chevron structure were recently predicted to have a negative Poisson's ratio in a specified direction, the physical mechanism of which was illustrated by a hinged framework that unfolds under tension $[12,13]$, whereas the natural hierarchical laminates were deduced to rarely have such a characteristic [14,15]. However, the natural hierarchical laminates such as the nacres of molluscs have a fracture work 3 to 4 orders of magnitude higher than the pure mineral made of the materials themselves $[16,17]$, to which synthetic hierarchical laminates are never comparable $[18,19]$. It is implied that nacre has the capacity of energy absorption much higher than that of the synthetic laminates. Many investigations proved that the microstructure of nacre was the main reason producing the high mechanical performance of the material [20-28], so that the microstructure has become the biomimetic design target of synthetic high performance materials [29-33]. Although the synthetic materials that mimicked nacre's microstructure were proven to be more fracture resistant than the materials without the microstructure, they did not have a toughness comparable to nacre [19]. This is an indication that the microstructural mechanisms generating the excellent mechanical properties of nacre have not been well understood yet [28-31]. In the present Letter, we first reveal a novel phenomenon in the tension deformation of nacre, i.e., the effect of the negative Poisson's ratio of about -0.38 ; then, we indicate that the effect can yield very high energy absorption so that the material obtains a tensile deformation about 1 order of magnitude greater than that of the aragonite mineral made of nacre itself, and finally, the mechanism yielding the negative Poisson's ratio, which is proven to be different at all from the reported previously, is pointed out.

Test samples were made of the nacre of the Hyriopsis schlegeli shells and were soaked in water over three weeks. We performed in situ quasistatic uniaxial tension tests of nacre in a Hitachi S-570 scanning electron microscope (SEM) equipped with a loading stage, which was operated at a strain rate $0.001 \mathrm{~s}$ up to failure. All tested data of longitudinal stress-strain, together with the surface images of nacre at different loading levels, were automatically recorded by the SEM and dealt with by using the image analysis system. Based on the surface images, we used the digital speckle correlation method to obtain the longitudinal and transverse strain fields of nacre. In the tensile tests, the behavior of nacre initially presented a linear elastic response such as a pure aragonite mineral when longitudi- 
nal strain was less than $1.2 \times 10^{-3}$. Then, a large region of inelastic strain, which coarsely ranged from $1.2 \times 10^{-3}$ to 0.01 and appeared to harden slightly, was yielded from elastic limit stress of about $70 \mathrm{MPa}$ up to the failure of nacre, as shown in Fig. 1(a). In the elastic deformation region, the transverse strain of nacre was approximately measured to be about $-0.4 \times 10^{-3}$, as shown in Fig. 1(b), and the Poisson's ratio of nacre was calculated as 0.32 , which is very similar to aluminum. In the inelastic strain region, when the longitudinal strain increased from $1.2 \times$ $10^{-3}$ to $4.4 \times 10^{-3}$, the tested sample continued to contract laterally up to the transverse strain approaching to $-1.71 \times 10^{-3}$. The average Poisson ratio in this region was approximated to be 0.42 , which is similar to lead and

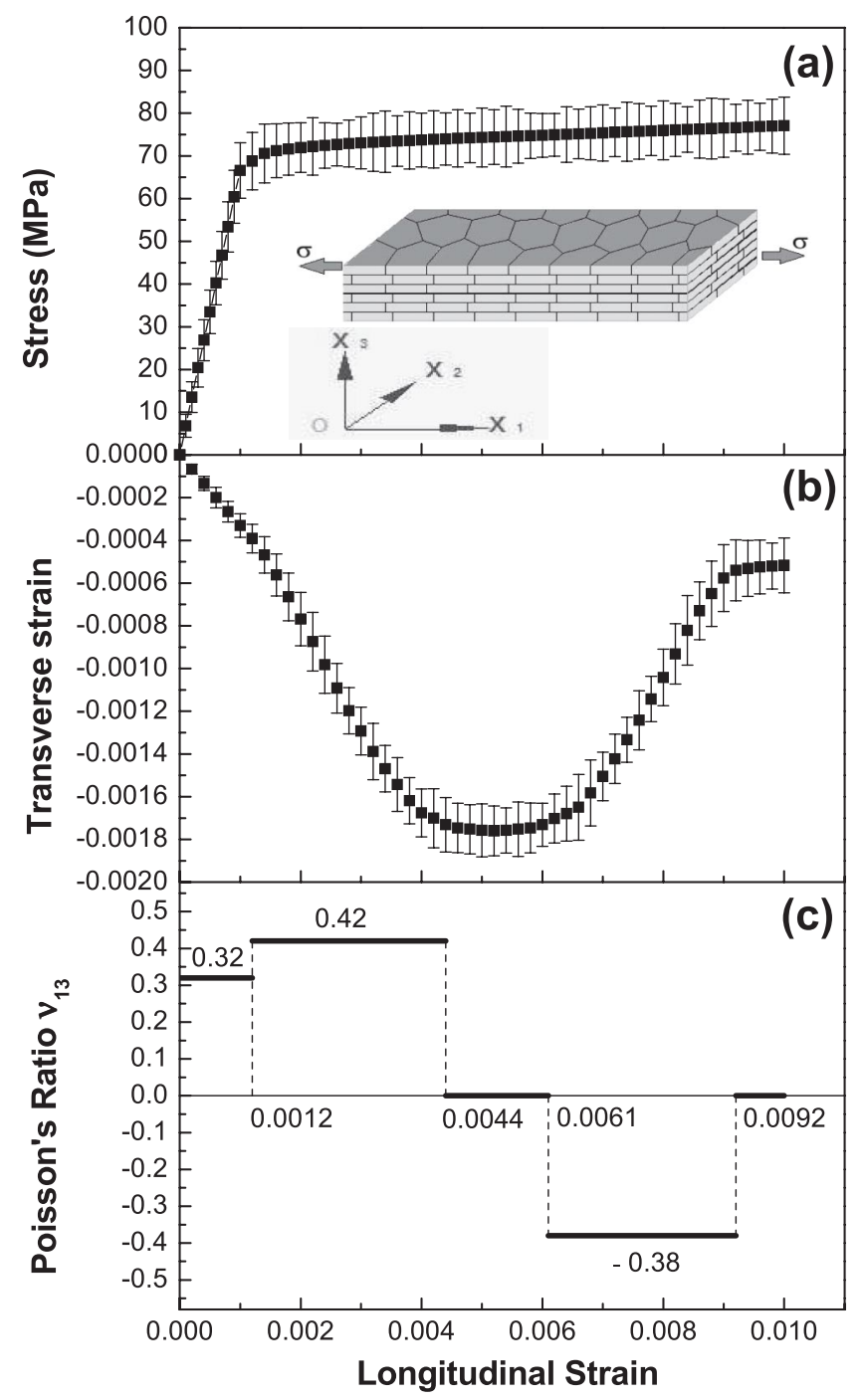

FIG. 1. (a) Tension test curve and direction of nacre. (b) Curve of longitudinal-transverse strains of nacre. (c) The changes of Poisson's ratio $\nu_{13}$ in the deformation of nacre, where the first index stands for the direction of applied load $x_{1}$ and the second index stands for the direction of transverse deformation $x_{3}$, as shown in the inset of (a). rubbers; when the longitudinal strain increased from $4.4 \times$ $10^{-3}$ to $6.1 \times 10^{-3}$, the transverse contraction of nacre ceased and the transverse strain coarsely maintained a constant, $-1.71 \times 10^{-3}$, and the average Poisson's ratio approximated to be zero, which is coarsely equal to cork; however, when the longitudinal strain was increased from $6.1 \times 10^{-3}$ to $9.2 \times 10^{-3}$, the tested sample appeared to undergo a transverse expansion. In this deformation region, the total value of transverse strain was increased from $-1.71 \times 10^{-3}$ to about $-0.54 \times 10^{-3}$ and the corresponding transverse strain was measured to be $+1.17 \times 10^{-3}$. The Poisson's ratio of nacre became a negative value and was computed to be about -0.38 , which is a novel value of Poisson's ratio that has not been found in existing materials, and when the longitudinal strain was greater than $9.2 \times$ $10^{-3}$ and up to the failure of the sample, the transverse strain remained a constant and the Poisson's ratio of nacre approximated that of cork again, as shown in Figs. 1(b) and 1(c). In the whole tension deformation, the total longitudinal and transverse strain of nacre were measured to be about $\varepsilon_{L}=0.01$ and $\varepsilon_{T}=-0.54 \times 10^{-3}$, respectively.

In the deformation that nacre experienced, the longitudinal strain with the transverse strain region of a negative Poisson's ratio was approximately equal to that with the transverse strain region of a positive Poisson's ratio. Compared with ordinary materials, the effect of the negative Poisson's ratio should play a key role in the large deformation that nacre generated in the tension. Under an applied stress $\sigma$, the total strain energy per unit volume of the tested material is written as $U=\sigma^{2} / 2 E$, which can be separately divided into the density of volume strain energy, $U_{V}=(1-2 \nu) \sigma^{2} / 6 E$, and the density of deformation strain energy, $U_{D}=(1+\nu) \sigma^{2} / 3 E$ [34], where $\nu$ and $E$ are the Poisson's ratio and the Young's modulus of material, respectively. When the tested nacre transversely shrank with the Poisson's ratio $\nu=0.42$, the ratio between the densities of volume and total strain energy was calculated to be $U_{V 1} / U \approx 5.3 \%$ and the ratio between the densities of deformation and total strain energy was expressed as $U_{D 1} / U \approx 94.7 \%$. However, when the tested nacre transversely expanded with the Poisson's ratio $\nu=$ -0.38 , the ratio between the densities of volume and total strain energy was $U_{V 2} / U \approx 58.7 \%$ and the ratio between the densities of deformation and total strain energy was $U_{D 2} / U \approx 41.3 \%$. It is shown that the negative Poisson's ratio largely increased the density of volume strain energy and effectively decreased the density of deformation strain energy in the total energy that nacre was input in the tension. In particular, compared the state of strain energy under $\nu=-0.38$ with that under $\nu=0.42$, we obtain the ratios between the two volume strain energies and between the two deformation strain energies to be $U_{V 2} / U_{V 1}=11$ and $U_{D 2} / U_{D 1} \approx 0.44$, respectively. This is an indication that the effect of the negative Poisson's ratio effectively increased the volume strain energy per unit volume of the 
material by $1100 \%$ and decreased the deformation strain energy per unit volume of the material by about $44 \%$. It is implied that nacre spent more energy on its volume change and less energy on its deformation. Therefore, the materials with a negative value of Poisson's ratio, compared with ordinary materials, can absorb more input work before the failure of the materials.

In order to study the mechanism generating the negative Poisson's ratio in the tensile tests of nacre, we based on the microstructures of nacre, together with the fact that the deformation and failure of nacre only occurs in the overlap region of each of the aragonite platelets of nacre when nacre is subjected to a tensile stress in the direction parallel to the aragonite platelets $[25,27,28]$. Nacre is renowned for the "brick-mortar" microstructure [16,30], which contains 95 vol.\% of interlocking aragonite platelets staggered in successive layers and separated by $5 \%$ organic matrix layers in which there are large numbers of mineral bridges passing through the mortar layers and connecting successive aragonite platelets [35-37], as shown in Fig. 2. From the microstructures of nacre, we calculated the average sliding distance of an overlap region in the tension to be $\Delta l=\varepsilon_{L} \times l_{p}=80 \mathrm{~nm}$, which is equal to the average spacing between two neighboring mineral bridges in the overlap region, where $l_{p}=8 \mu \mathrm{m}$ is the average spacing of each of the aragonite platelets $[20,36]$. This is an indication that the whole course of the tension of nacre just corresponds to the course that a mineral bridge in the overlap region experienced from shear deformation and fracture, sliding in the organic matrix layer and barging up against a neighboring mineral bridge, up to the climbing between the two neighboring mineral bridges on one another and finally shearing to fracture the macromolecules of the organic matrix, as shown in Figs. 3(a)-3(e). In detail, when the longitudinal strain of nacre was from 0 to $4.4 \times 10^{-3}$, the mineral bridges in the overlap region deformed and fractured, and then, the upside and the underside of each of the fractured mineral bridges intrude into the organic matrix in the opposite direction. In this course, the mineral bridges in the overlap region were rapidly fractured one by one and the macromolecules of organic matrix became the principal carrier of the load in the overlap region [27,28]. When the longitudinal strain of the tested nacre ranged from $4.4 \times 10^{-3}$ to $6.1 \times 10^{-3}$, the upside and the underside of each of the fractured mineral bridges levelly slid along the opposite direction in the organic matrix layer. When the longitudinal strain ranged from $6.1 \times 10^{-3}$ to $9.2 \times 10^{-3}$, the different rest parts of the two neighboring fractured mineral bridges climbed one another. It is such the climbing that nacre produces transverse expansion and negative Poisson's ratio in the tension deformation process, which is different at all from the mechanism of the man-made laminates by hinged framework that unfold under tension $[12,13]$ and the twinned crystal of cubic metals [7-9]. Finally, the climbing sheared to fracture all macromolecules of organic matrix and resulted in the failure of nacre.
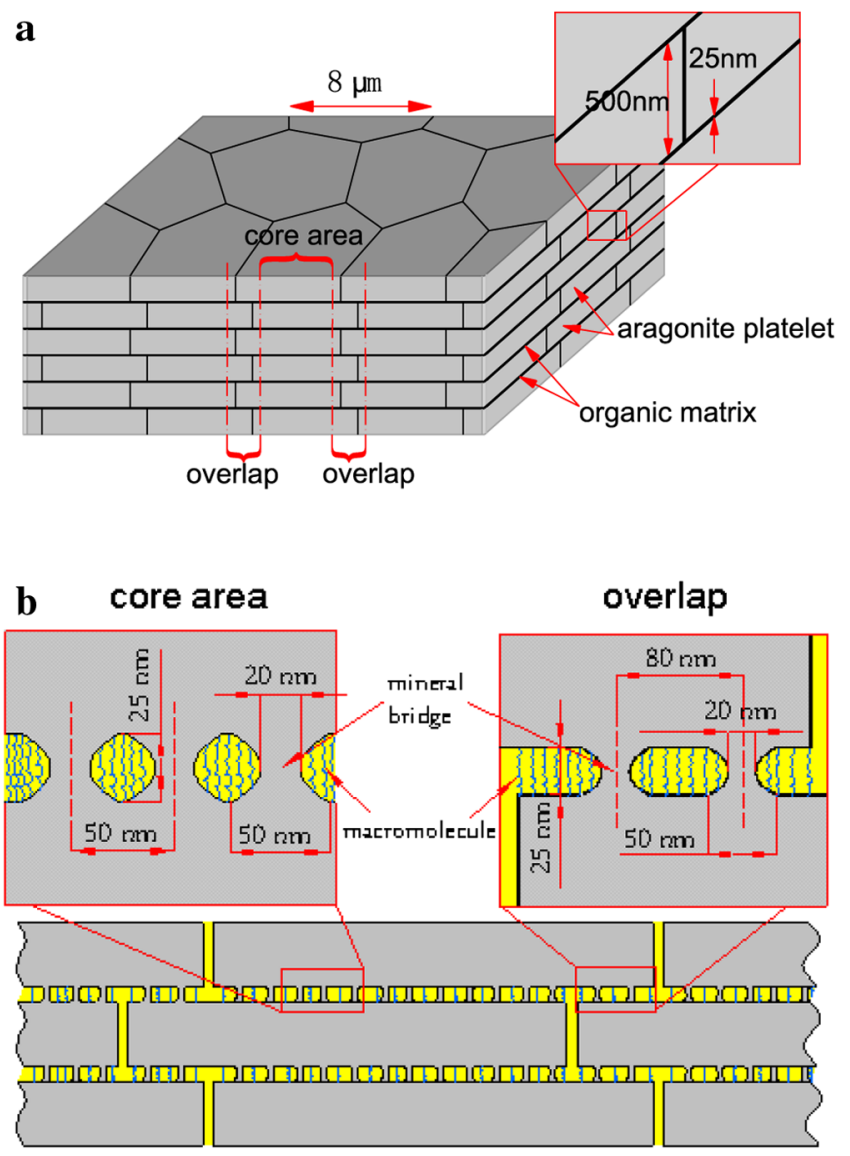

FIG. 2 (color online). Schematic illustrations showing the microarchitecture of nacre. (a) The average thickness of an aragonite platelet layer and an organic matrix layer are 500 and $25 \mathrm{~nm}$, respectively, the average spacing of each of the aragonite platelets is about $8 \mu \mathrm{m}$. An aragonite platelet of nacre is divided into two regions: The "core" is the region that is common to the platelets belonging to the same column, and the "overlap" region corresponds to the area where platelets from adjacent columns overlap. The length of overlap is about $1 / 3$ length of a platelet on the cross section of nacre. (b) There are a large number of mineral bridges in the organic matrix layers. The minimum and maximum parts of the diameter of the mineral bridges are about 20 and $50 \mathrm{~nm}$, respectively, and the height of the mineral bridges is equal to the thickness of the organic matrix layers. In the core region, the average bridge-to-bridge spacing between the neighboring mineral bridges is about $50 \mathrm{~nm}$ and in the overlap region, the average bridge-to-bridge spacing between the neighboring mineral bridges is about $80 \mathrm{~nm}$.

As stated above, the effect of a negative Poisson's ratio can effectively enhance the energy absorption and the deformation capability in the deformation of materials, which is vital to the applied processes of a lot of engineering materials, especially engineering ceramics. Therefore, the microstructures that can induce to yield the effect of a negative Poisson's ratio should be given sufficient attention in the engineering design of synthetic composites. 


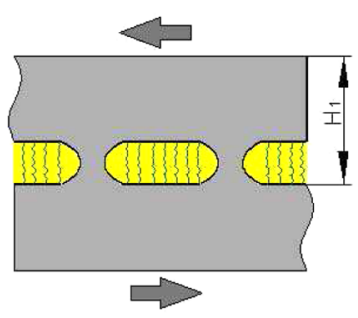

a
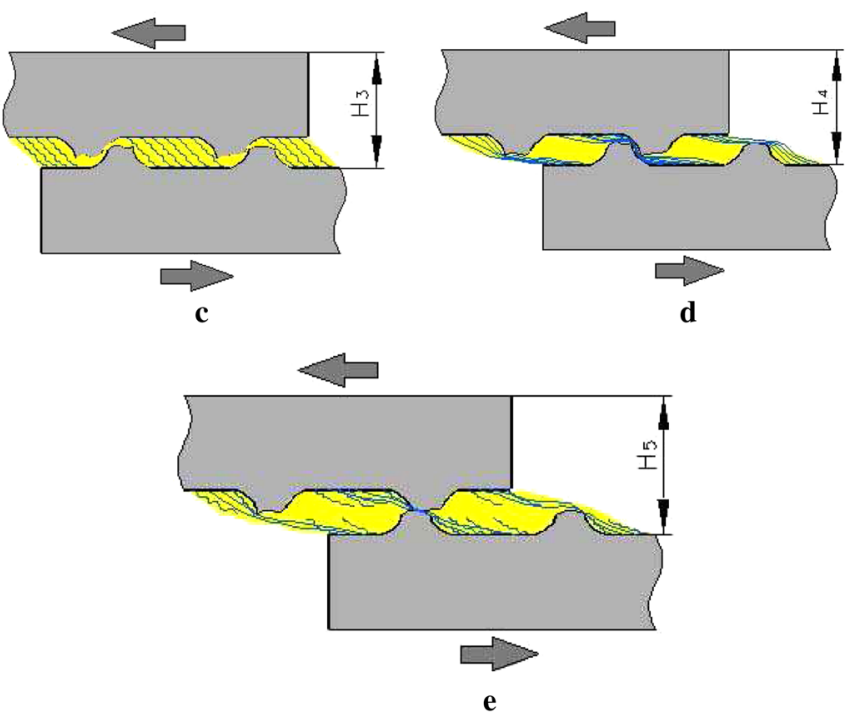

FIG. 3 (color online). Schematic illustrations showing the deformation between two neighboring mineral bridges in the overlap region of an organic matrix layer of nacre. (a) Both the mineral bridges and the organic matrix are applied to a shear stress and nacre laterally contracted in elastic. At this time, the thickness of the organic matrix and aragonite platelet is about $H_{1} \approx 524.79 \mathrm{~nm}$. (b) The neighboring mineral bridges fracture, $H_{2} \approx 524.79 \mathrm{~nm}$. (c) The rest parts of the two fractured mineral bridges intrude into the organic matrix and start to slide along the opposite direction in the organic matrix layer, $H_{3} \approx 524.10 \mathrm{~nm}$. (d) The rest parts of the two neighboring fractured mineral bridges slide to meet and begin to climb one another, $H_{4} \approx$ $524.10 \mathrm{~nm}$. (e) The rest parts of the two neighboring fractured mineral bridges climb one another to arrive at the tops and shear to fracture in sequence the macromolecules of the organic matrix, $H_{5} \approx 524.72 \mathrm{~nm}$.

This work was supported by the National Natural Science Foundations of China (Grants No. 10732050, No. 10721202) and CAS Innovation Program (Grant No. KJCX2-YW-M04).

*To whom all correspondence should be addressed. songf@lnm.imech.ac.cn
[1] S. P. Timoshenko and J. N. Goodier, Theory of Elasticity (McGraw-Hill, New York, 1970), 3rd ed.

[2] Y.C. Fung, Foundations of Solid Mechanics (PrenticeHill, Englewood Cliffs, NJ, 1968).

[3] R. Lakes, Science 235, 1038 (1987).

[4] J. J. Lewandowski, W.H. Wang, and A.L. Greer, Philos. Mag. Lett. 85, 77 (2005).

[5] P. M. Singh and J. J. Lewandowski, Scr. Metall. Mater. 29, 199 (1993).

[6] P. M. Singh and J. J. Lewandowski, Metall. Mater. Trans. A 26, 2911 (1995).

[7] A.E. H. Love, A Treatise on the Mathematical theory of Elasticity (Dover, New York, 1944), 4th ed.

[8] A. Y. Haeri, D. J. Weidner, and J. B. Parise, Science 257, 650 (1992).

[9] R. H. Baughman et al., Nature (London) 392, 362 (1998).

[10] J. B. Choi and R. Lakes, Int. J. Fract. 80, 73 (1996).

[11] X. Shang and R. Lakes, Phys. Status Solidi (b) 244, 1008 (2007).

[12] L. J. Gibson et al., Proc. R. Soc. A 382, 25 (1982).

[13] R. Lakes, Nature (London) 361, 511 (1993).

[14] L. Rothenburg, A. A. Berlin, and R. J. Bathurst, Nature (London) 354, 470 (1991).

[15] G. Milton, J. Mech. Phys. Solids 40, 1105 (1992).

[16] J. D. Currey, Proc. R. Soc. B 196, 443 (1977).

[17] A. P. Jackson, J. F. V. Vincent, and R. M. Turner, Proc. R. Soc. B 234, 415 (1988).

[18] B. L. Smith et al., Nature (London) 399, 761 (1999).

[19] N. Almqvist et al., Mater. Sci. Eng., C 7, 37 (1999).

[20] F. Song, A.S. Soh, and Y. L. Bai, Biomaterials 24, 3623 (2003).

[21] R. Menig et al., Acta Mater. 48, 2383 (2000).

[22] H. Gao et al., Proc. Natl. Acad. Sci. U.S.A. 100, 5597 (2003).

[23] C. Kearney et al., Phys. Rev. Lett. 96, 255505 (2006).

[24] X. Li et al., Nano Lett. 4, 613 (2004).

[25] R. Z. Wang et al., J. Mater. Res. 16, 2475 (2001).

[26] K. Okumura and P. G. de Gennes, Eur. Phys. J. E 4, 121 (2001).

[27] F. Barthelat et al., J. Mech. Phys. Solids 55, 306 (2007).

[28] F. Barthelat and H.D. Espinosa, Exp. Mech. 47, 311 (2007).

[29] M. A. Meyers et al., JOM 58, 36 (2006).

[30] L. Addadi and S. Weiner, Nature (London) 389, 912 (1997).

[31] M. Rubner, Nature (London) 423, 925 (2003).

[32] M. Sarikaya, Proc. Natl. Acad. Sci. U.S.A. 96, 14183 (1999).

[33] Z. Tang et al., Nat. Mater. 2, 413 (2003).

[34] E. J. Hearn, Mechanics of Materials (Pergamon, Oxford, 1977).

[35] T. E. Schaffer et al., Chem. Mater. 9, 1731 (1997).

[36] F. Song, X. H. Zhang, and Y.L. Bai, J. Mater. Res. 17, 1567 (2002).

[37] A. Lin and A. M. Meyers, Mater. Sci. Eng. A 390, 27 (2005). 